\title{
Comparison of Indirect Immunofluorescence Assay as a Confirmatory Test to Detect Antibody to Human Immunodeficiency Virus with Line Imuune Assay and Western Blot Methods
}

\author{
Ahmed Nawsher Alam, ${ }^{1,3}$ Mahmuda Siddiqua, ${ }^{2}$ Shahina Tabassum, ${ }^{1}$ Md Nazrul Islam ${ }^{1}$ \\ 'Department of Virology, Bangabandhu Sheikh Mujib Medical University, Shahbag, Dhaka; 2Department of Microbiology, Ibn-Sina \\ Medical College, Dhaka; ${ }^{3}$ Currently, Virology Section, Microbiology Laboratory, Institute of Public Health, Mohakhali, Dhaka
}

\begin{abstract}
In low prevalent countries of Human Immunodeficiency Virus (HIV) like Bangladesh, it is recommended that all HIVpositive sera tested by Enzyme Linked Immunosorbent Assay (ELISA) and/or rapid tests should be confirmed by Line Immune Assay (LIA) or Western Blot (WB) method. As these two tests are quite expensive, Indirect Immunofluorescence Assay (IFA) was evaluated as a confirmatory test and as an alternative to these two methods in the present study. A total of 92 subjects consisting of $46 \mathrm{HIV}$-antibody-positive patients and 46 controls were included in the study. All samples of sera were tested by ELISA and IFA methods, and some 34 of 46 HIV-antibody-positive samples were tested by LIA. One ELISA positive serum was found negative by both IFA and LIA. This result indicates that ELISA was $\mathbf{1 0 0} \%$ sensitive and $\mathbf{9 8 . 7} \%$ specific for detection of HIV antibody. Comparison between LIA with IFA on 34 samples, and WB with IFA on 26 samples showed $100 \%$ correlation between these methods. The study concluded that the IFA method is equal in performance as LIA and WB methods for the detection of antibody to HIV and can be used as a confirmatory test.
\end{abstract}

Key words: Human Immunodeficiency Virus, Indirect Immunofluoroscence Assay, Line Immuno Assay, Western Blot, Capillus (rapid test)

\section{Introduction}

The total number of people living with the Human Immunodeficiency Virus (HIV) has reached its highest level and an estimated 40.3 million (36.7-45.3 million) people are now living with HIV. ${ }^{1}$

Acquired Immunodeficiency Syndrome (AIDS) has killed more than 25 million people since it was first recognized in 1981, making it one of the most destructive epidemics in the recorded history. Despite recent improved access to anti-

Correspondence:

Dr. Ahmed Nawsher Alam

Bacteriologist, Institute of Public Health

Mohakhali, Dhaka-1212

E-mail: nawsher_nfs@yahoo.com retroviral treatment (ART) and care in many regions of the world, the AIDS epidemic claimed 3.1 million lives in 2005 of which more than half a million were children. ${ }^{1}$

Transmission of HIV infection has been documented to occur through one or more of the four modes: sexual contact, exposure to blood-contaminated needles, administration of infected blood or blood products, and passage of virus from infected mother to their newborn babies. ${ }^{2}$ As the prevalence of HIV infection increases, the general population will be at greater risk, primarily through heterosexual transmission. ${ }^{3}$ The fear that HIV infection could be transmitted by blood transfusion, prompted the development of a diagnostic routine screening test for HIV antibodies. ${ }^{4}$ In addition to ensuring safe blood transfusion, the test has been useful for other prevention efforts in high-risk groups and in making 
diagnosis of HIV infected patients.

Several types of assays have been developed for detection of antibody to HIV including Enzyme Immunoassays (EIAs) with whole virus antigen or recombinant antigens, Western Blot (WB) analysis, Indirect Immonufluorescence Assay (IFA), Radioimmunoprecipitation Assay (RIPA), Immune peroxidase test, Neutralizing test (Nt), Latex Agglutination test, and Passive Particle Agglutination (PPA) test.4-14 The EIA is the most widely used serological test for HIV antibody detection in the world today. Because EIA is very sensitive, very specific, fast and less expensive, it is used primarily as the screening test of choice. However, if a specimen is found repeatedly reactive with a screening test, it is re-tested with a supplemental assay such as WB, IFA or RIPA. Positive reactivity by one of these supplemental assays confirm the presence of HIV antibody. ${ }^{15}$

Another very promising test is Line Immune Assay (LIA), the principle of which is similar to the WB. ${ }^{16}$ Both the tests have several disadvantages namely high cost, variability in quality of strips, difficulty in defining minimum criteria for a positive result. ${ }^{17}$ In contrast, IFA offers many practical advantages as a validating test for ELISA. The IFA is a routine test being familiar to trained clinical laboratory personnel. The test can be performed in less than 2 hours whereas LIA and WB require overnight incubation. The IFA is a less expensive method for HIV serology and can be used as screening as well as a confirmatory test.7,18,19

Considering the above aspects of detection of HIV anitbody, the current study was designed to evaluate utility of the Indirect Immunofluorescence Assay (IFA) as a confirmatory test to detect HIV antibody.

\section{Methods}

This study was conducted in the department of Virology, Bangabandhu Sheikh Mujib Medical University (BSMMU), Dhaka from July, 2005 to December, 2006. A total of 92 subjects were selected for the study of whom 46 were HIVantibody-positive patients and 46 were controls. The HIVantibody-positive patients were divided into two groups for the purpose of the study: Group I- consisting of 20 samples positive by screening tests (ELISA and Capillus), and Group II- comprising of 26 samples taken from preserved samples, which were kept at $-20^{\circ} \mathrm{C}$ in Virology laboratory of the BSMMU. The preserved samples were previously positive by
ELISA and confirmed by western blot (WB) method. During the study, all $46 \mathrm{HIV}$-antibody-positive samples including groups I and II were tested by ELISA (re-tested for confirmation), LIA and IFA methods.

The controls comprised of different groups including healthy controls $(n=16)$, patients with autoimmune disease like Systemic Lupus Erythematosus and Rheumatoid Arthritis $(\mathrm{n}=10)$, multiple blood-transfused patients like Thalassaemia and Leukaemia $(\mathrm{n}=10)$, and multipara pregnant women $(n=10)$. The control groups were also tested by ELISA and IFA methods.

\section{Enzyme Linked Immunosorbent Assay (ELISA)}

By principle of the test, antibodies present in patient's serum react with antigens coated into microtiter plate. The antigens comprise of 3 recombinant (rec) proteins gp41, gp36 and p24 and peptide gp41. When antibodies against HIV-1 or HIV-2 were present in the test serum, the antibodies bound to the microplate. Any non-specifically bound material was removed by washing procedure and the antigen-conjugate was then added. The conjugate is a mixture of recombinant proteins and peptides from the Gag/Env regions of the HIV-1 and HIV-2 which are directly labelled with Horse Raddish Peroxidase. Unbound enzyme conjugate was removed in a further washing step. The presences of bound antibodyantigen complexes were demonstrated using enzyme reaction with Tetramethyl Benzidine (TMB) as a substrate, which resulted in a coloured product. The enzyme reaction was stopped using Sulphuric Acid and reading was taken by ELISA reader (Organo Teknika Microwell System, Germany) at $450 \mathrm{~nm}$.

\section{Indirect Immunofluorescence Assay (IFA)}

For Indirect Immunofluorescence Assay (IFA) procedure, Fluorognost HIV-1 IFA kit (Sanochemia Inc., USA) was used. In the test procedure, HIV-infected and uninfected Tcells were applied to the well of fluorescence slide to make cell smears. Here, infected PALL T-cells and un-infected PALL T-cells were used in the glass slides of the kit to make cell smears. These cell smears were then air-dried, fixed in Acetone for 10 minutes and stored at $-20^{\circ} \mathrm{C}$ or below. During the assay, slides were warmed to room temperature, diluted test serum was applied to each well of the slide and incubated. After washing, Fluorescein Isothiocyanate conjugated anti-human IgG was applied to each well and incubated. Slides were then washed, dried and mounted in glycerol, and finally read under fluorescence microscope (Olympus, Japan; model- 209). 


\section{Line Immune Assay (LIA)}

Test samples were incubated in a test trough together with multiple antigen coated test strips (recombinant proteins and synthetic protein from HIV-1 and HIV-2 and a synthetic peptide from HIV-1 group $\mathrm{O}$ is coated as discrete lines as nylon strip with plastic backing). HIV antibodies, if present in the sample, bound to the individual HIV antigen lines on the strip. Then, a Goat anti-human IgG labelled with Alkaline Phosphatase was added, which was bound to any HIV antigen /antibody complex previously formed. Incubation with enzyme substrate (5-bromo-4-chloro-3-indolyl phosphate/ Nitroblue tetrazolium, BCIP/ NBT Alkaline Phosphatase Substrate Solution) produced a dark brown colour in proportion to the amount of HIV-antibody present in the sample. Colour development was stopped with 0.1 $\mathrm{mol} / \mathrm{L}$ Sulphuric Acid. In a negative sample, containing no HIV specific antibodies, the labelled anti-human antibody could not bind to antigen/ antibody complex, and only a low standard background colour developed.

\section{Results}

All samples (46) from the study subjects were found positive by repeat Enzyme Linked Immunosorbent Assay (ELISA). But 45 samples (19 from group I and 26 from group II) were positive by Indirect immunofluorescence Assay (IFA) and the remaining one sample from group I was negative by the test. None of the control sera tested was positive for HIV antibody. (Table I)

Table I: Results of ELISA and IFA in HIV antibody positive patients and controls

\begin{tabular}{lccccc}
\hline Name of Groups & No. of & \multicolumn{2}{c}{ ELISA (repeat) } & \multicolumn{2}{c}{ IFA } \\
& test done & +ve & -ve & +ve & -ve \\
\hline Group I & 20 & 20 & 00 & 19 & 01 \\
Group II & 26 & 26 & 00 & 26 & 00 \\
Healthy control & 16 & 00 & 16 & 00 & 16 \\
$\begin{array}{l}\text { Auto immune disease } \\
\text { Multiple blood }\end{array}$ & 10 & 00 & 10 & 00 & 10 \\
transfused & 10 & 00 & 10 & 00 & 10 \\
$\begin{array}{l}\text { Multipara } \\
\text { pregnant women }\end{array}$ & 10 & 00 & 10 & 00 & 10 \\
Total & 92 & 46 & 46 & 45 & 47 \\
\hline
\end{tabular}

Comparison between ELISA and IFA results showed that all $(n=92)$ but one specimen had same result by both ELISA and
IFA methods. One ELISA positive sample was negative by IFA. So, the calculated sensitivity of ELISA was $100 \%$ and specificity $97.8 \%$. Comparison of Western Blot (WB) and IFA results showed that in group II, all 26 WB-positive samples were again found positive by IFA method. So, the sensitivity of IFA was $100 \%$. (Table I)

Comparison between IFA and Line Immune Assay (LIA) on 34 HIV-antibody-positive sera showed that 33 were positive and 1 serum was negative by both the methods. So, the sensitivity and specificity of IFA was $100 \%$. (Table II)

Table II: Comparison between LIA and IFA test results of 34 HIV positive patients

\begin{tabular}{lccc}
\hline LIA & \multicolumn{3}{c}{ IFA } \\
Positive & Negative & Total \\
\hline Positive & 33 & 00 & 33 \\
Negative & 00 & 01 & 01 \\
Total & 33 & 01 & 34 \\
\hline
\end{tabular}

For IFA method: calculated sensitivity $=100 \%$, and specificity $=100 \%$

\section{Discussion}

This study was conducted to assess the appropriateness of Indirect Immunofluorescence Assay (IFA), which is less costly and takes less time. A study from India found the cost of testing by IFA $20 \%$ less than that of Western blot (WB) analysis. ${ }^{17}$ However, IFA needs fluorescence microscope, which involves initial establishment, costing more than the WB or Line Immune Assay (LIA). But in laboratories where fluorescence microscope is already available, the IFA can be an inexpensive method of confirming the presence of HIV antibody.

In the present study, WB positive samples were tested by IFA for comparison. All 26 sera (group II) were also found positive by IFA method giving a $100 \%$ correlation between these two methods. There were excellent agreements in terms of sensitivity and specificity between IFA and WB in some previous studies. In a study, all 60 samples from 142 homosexual men were positive by Enzyme Immuno Assay (EIA), IFA and WB showing $100 \%$ agreement among them. ${ }^{7}$ Another study from the USA demonstrated $100 \%$ agreement between IFA and WB on testing 271 sera from high risk group. ${ }^{18}$ One study in Massachusetts General Hospital tested 104 sera by IFA and WB and found all 68 WB-negative sera also negative by IFA. One of the $36 \mathrm{WB}$-positive samples was 
found negative by IFA, and providing sensitivity and specificity of $97.22 \%(35 / 36)$ and $100 \%$ respectively. ${ }^{9}$ Among 156 sera, investigated by Johnson et al (1988), 18 were equivocal either by IFA or WB or both, allowing direct comparison of 138 specimens. Sensitivity of IFA compared to WB was $99.2 \%$ and specificity $100 \% .{ }^{20}$ Abraham et al (1994) in India demonstrated that among 42 WB-positive specimens, 41 were IFA positive, showing sensitivity of $97.6 \%$. Of the 46 WB-negative samples, 45 were negative by IFA showing the specificity of $97.8 \% .{ }^{17}$

In the present study, LIA was done on 34 out of 46 HIVpositive samples due to reagent constraint. These samples were also tested by IFA. Comparison between these two test methods showed $100 \%$ correlation in terms of sensitivity and specificity. It was also observed that all the cases were positive for HIV-1 antibody, though antigens for both HIV-1 (gp120 and gp 41) and HIV-2 (gp 105 and gp 36) were fixed on the nitrocellulose strips. The present study indicated that none of the $33 \mathrm{HIV}$-positive samples tested by LIA was due to HIV-2 infection. All male subjects positive for HIV antibody in this study were migrant workers from the Middle East, Malaysia, Singapore and Thailand, where HIV-1 is prevalent. None of them was from Africa, where HIV-2 is present, probably because of this geographical distribution of the types, no HIV-2 was detected in this study.

In the present study, one sample positive for HIV antibody from "Group I," was found positive again when re-tested by ELISA. However, this sample was found negative when tested by IFA and LIA methods. This indicates that even the results obtained by the well-known 3-tests algorithm of highly sensitive and specific methods may sometimes show false positive results. So, all the positive sera found by the 3-tests algorithm should be confirmed by a supplementary test like LIA and IFA, especially in low-prevalent countries like Bangladesh.

In the present study, no false positive or non-specific result was seen by IFA method in HIV-antibody-positive patients as well as in control groups. Similarly, no false positive or nonspecific result was observed by IFA method in other studies. ${ }^{19}$ Non-specific reaction may occur due to formation of antiHLA antibodies, anti-nuclear antibody and anti-lymphocyte antibodies. ${ }^{18,20}$ In the present study, none of the control subjects showed any positive result with IFA. As such, it is suggested that WB and LIA can be replaced by IFA as a confirmatory test for HIV-antibody detection. However, further extensive studies should be done to achieve dependable conclusion.

\section{Acknowledgement}

Fluorognost IFA HIV-1 test kits used in this study was supplied by Mr. RC Stephens, General Manager, Sanochemia Inc., USA free of cost.

\section{References}

1. United Nations programme on HIV/AIDS, World Health Organization. Introduction. In: AIDS Epidemic Update, December, 2005. Geneva, Switzerland: UNAIDS/WHO; 2005: p.2.

2. Curran JW. The Epidemiology and Prevention of Acquired Immunodeficiency Syndrome. Ann Intern Med 1984; 103: 657662.

3. Quinn TC, Mann JM, Curran JW, Piot P. AIDS in Africa: An Epidemiologic Paradigm. Science 1986; 234: 955-963.

4. Sarngadharan MG, Popovic M, Bruch L, Schupbach J, Gallo RC. Antibodies reactive with human $\mathrm{T}$ lymphotropic retroviruses (HTLV-III) in the serum of patients with AIDS. Science 1984; 224: 506-508.

5. Smith RS, Naso RB, Rosen J, Whalley A, Hom YL, Richman K. Antibody to a synthetic oligopeptide in subjects at risk for human immunodeficiency virus infection. J Clin Microbiol 1987; 25: 1498-1504.

6. Thorpe R, Brasher MDR, Bird CR, Garrett AJ, Jacobs AJ, Schild GC. An improved immunoblotting procedure for the detection of antibodies against HIV. J Virol Methods1987; 16: 87-96.

7. Gallo D, Diggs JL, Shell GR, Dailey PJ, Hoffman MN, Riggs JL. Comparison of detection of antibody to the acquired immune deficiency syndrome virus by enzyme immunoassay, immunofluorescence, and Western blot methods. J Clin Microbiol 1986; 23: 1049-1051.

8. Kaminsky LS, McHugh T, Stites D, Volberding P, Henle G, Levy JA. High prevalence of antibodies to acquired immune deficiency syndrome (AIDS) associated retrovirus (ARV) in AIDS and related conditions but not in other disease states. Proc Natl Acad Sci 1985; 82: 5535-5539.

9. Sandstrom EG, Schooley RT, Ho DD, et al. Detection of human anti-HTLV-III antibodies by indirect immunofluorescence using 
fixed cells. Transfusion 1985; 25: 308-312.

10. Shah AJ, Farzadegan H, Fox R, Nishanian P, Rinaldo, Phair JP. Detection of early antibodies in human immunodeficiency virus infection by enzyme linked immunosorbent assay, Western blot, and radioimmunoprecipitation. J Clin Microbiol 1987; 25: 1605-1610.

11. Karps A, Gilson W, Bevan PC, Oates JK. Lytic infection by British AIDS virus and development of a rapid cell test for antiviral antibodies. Lancet 1985; 2: 695-697.

12. Prince A, Pascual MD, Kosolapov LB, Kuorokawa D, Baker L, Rubinstein P. Prevalence, clinical significance and strain specificity of neutralising antibody to the human immunodeficiency virus. J Infect Dis 1987; 156: 268-272.

13. Quinn TC, Riggin CH, Kline RL, Mulanga K, Sensionand MG, Fauci AS. Rapid latex agglutination assay using recombinant envelope polypeptide for the detection of antibody to the HIV. J Am Med Assoc 1988; 260: 510-513.

14. Yoshida T, Matsui T, Kobayashi S, Yamamoto N. Evaluation of passive particle agglutination test for the antibody to human immunodeficiency virus. J Clin Microbiol 1987; 25: 14331437.
15. Jackson JB, Balfour HH. Practical diagnostic testing for human immunodeficiency virus. Clin Microbiol Rev 1988; 1: 124-138.

16. Constantine NT, Zink H. HIV testing technologies after two decade evolution. Ind J Med Res 2005; 121: 519-538.

17. Abraham P, Babu PG, John TJ. Comparison of the indirect immunofluorescence assay with western blot for the detection of HIV antibody. Indian J Med Res 1994; 99: 143-148

18. Carlson JR, Yee J, Hinrichs SH, Byrant ML, Gardner MB, Pedersen NC. Comparison of Indirect Immunofluoresence and Western Blot for detection of anti human immunodeficiency virus antibodies. J Clinic Microbiol 1987; 25: 494-497.

19. Lennette ET, Karpatkin S, Levy JA. Indirect Immunofluorescence Assay for antibodies to Human Immunodeficiency Virus. J Clinic Microbiol 1987; 25: 199-202.

20. Johnson JE. Evaluation of a commercially developed indirect immunoflurescence system for the detection of anti human immunodeficiency virus antibodies. Diagn Microbiol Infect Dis 1988; 10: 159-163. 